\title{
Circulating and dynamic EMT
}

\section{CS \\ CTC}

characterization

might be

a useful

biomarker

for treatment response
Epithelial-mesenchymal transition (EMT) is thought to enable the dissemination of tumour cells into the surrounding tissue and circulation. However, characterizing the epithelial versus mesenchymal phenotypes of circulating tumour cells (CTCs) has been challenging because the expression of epithelial markers has typically been used as the basis for the isolation and visualization of CTCs from bulk blood samples. A new method to isolate and characterize both epithelial and mesenchymal CTCs has identified intriguing dynamics of EMT in CTCs from patients with breast cancer.

To distinguish epithelial from mesenchymal cancer cells, Shyamala Maheswaran, Daniel Haber and colleagues devised a method based on RNA in situ hybridization (RNA-ISH), in which cells are differentially stained according to the expression levels of seven epithelial genes versus three mesenchymal genes.

The authors first demonstrated the discriminating ability of the method on cell lines of known epithelial or mesenchymal phenotypes, both when cultured in vitro and in sections of mouse xenografts derived from these cells. Importantly, they also found that normal blood cells - which commonly copurify with cancer cells in CTC preparations were not stained. Furthermore, on sections of human breast cancer, they found a subpopulation of cells with epithelial morphology but which also had dual staining for epithelial and mesenchymal markers. These cells were more abundant in the aggressive cancer subtypes and might represent an intermediate state of EMT.

The authors then applied their RNA-ISH technique to CTCs in patients with breast cancer.
They used a microfluidic chip to immunoaffinity purify CTCs based on the expression of epidermal growth factor receptor (EGFR), ERBB2 and epithelial cell adhesion molecule (EPCAM).

CTCs were identified in the blood from 17 of 41 patients with different stages and subtypes of breast cancer. These CTCs were typically enriched for mesenchymal cells relative to the primary tumours, consistent with a role for EMT in tumour cell dissemination. Interestingly, in three patients, predominantly mesenchymal cells were found to form clusters. Although the relevance is currently unknown, this is contrary to the usual idea of EMT resulting in highly migratory single mesenchymal cells.

The authors also examined CTC dynamics during clinical responses to various treatments using paired pretreatment and post-treatment samples from ten patients, and seven serial samples from a further patient who underwent two rounds of treatment response and relapse. When treatment responses occurred, the expected decrease in total CTC counts was accompanied by a skew towards epithelial phenotypes, and these effects reversed during relapse in the serially sampled patient. Thus, CTC characterization might be a useful biomarker for treatment response. However, the extent to which this represents dynamic switching between epithelial and mesenchymal states, or a differential effect of treatment on existing epithelial and mesenchymal cancer cells, is currently unclear.

Finally, the authors used RNA sequencing of epithelial and mesenchymal CTCs to identify global gene expression signatures that are associated with breast cancer, EMT and therapeutic resistance. It will be interesting to fully assess the prognostic and predictive potential of various approaches to CTC characterization in breast cancer and other cancers.

Darren J. Burgess

ORIGINAL RESEARCH PAPER Yu, M. et al. Circulating breast tumor cells exhibit dynamic changes in epithelial and mesenchymal composition. Science 339, 580-584 (2013)

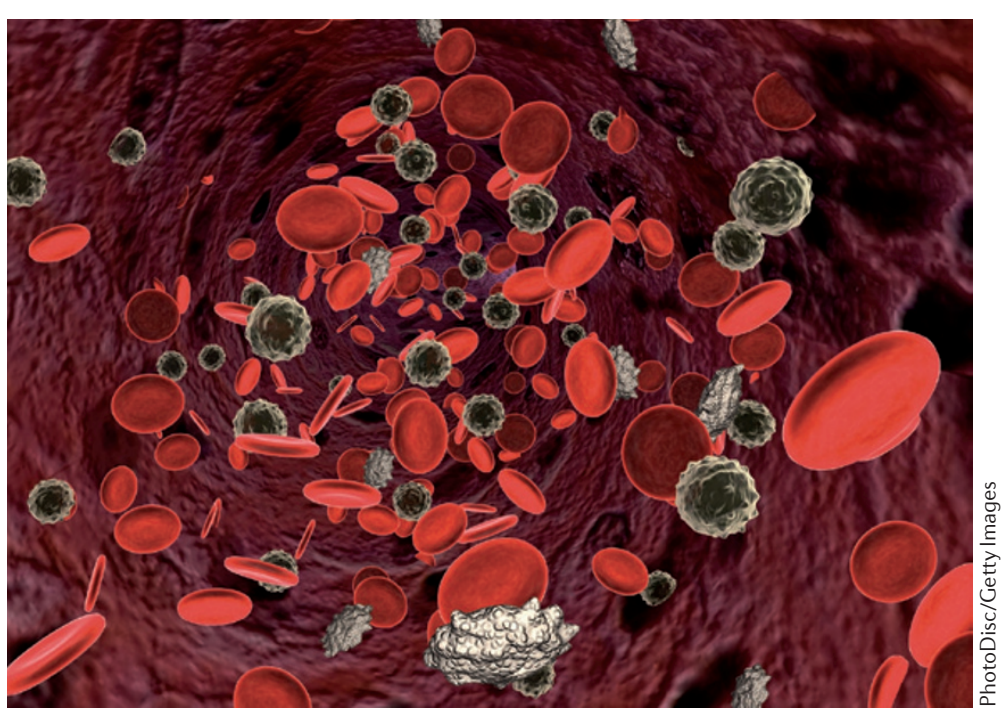

